\title{
THE RECORDING OF LATERAL POPLITEAL NERVE ACTION POTENTIALS IN MAN
}

\author{
BY
}

\author{
R. W. GILLIATT, H. V. GOODMAN, and R. G. WILLISON*
}

From the Institute of Clinical Research, the Middlesex Hospital Medical School, and the Department of Applied Electrophysiology, the National Hospital, Queen Square, London

It has been shown by Dawson and Scott (1949) that when the median or ulnar nerve is stimulated with a brief shock at the wrist an action potential can be recorded from the nerve at a higher level in the arm through electrodes placed on the skin over the course of the nerve. This technique has proved to be of considerable practical value in the assessment of patients with ulnar nerve lesions at the elbow (Gilliatt and Thomas, 1960).

The advantages of recording the nerve action potential are twofold. In the first place, the potential depends upon the passage of a synchronous volley of impulses under the recording electrodes, and it is thus highly sensitive to dispersion of impulses in different fibres of the nerve. In this way abnormalities of the nerve action potential may be detected when the degree of nerve damage is insufficient to affect conduction velocity in all the constituent fibres. The technique is thus more sensitive than conventional estimation of motor conduction velocity in which measurements of latency are made for the fastest surviving fibre. The second advantage of recording from the nerve itself is that impulses in sensory fibres make a substantial contribution to the action potential. The relative contributions of motor and sensory fibres vary in different nerves, but for the median and ulnar nerves it has been shown by Dawson (1956) that a substantial afferent volley in sensory fibres can be obtained by stimulation below or close to the motor threshold. Recording the nerve action potential may thus be expected to provide information about the behaviour of both motor and sensory fibres in a mixed nerve.

The work to be described in the present paper has been concerned with the recording of nerve action potentials from the lateral popliteal (peroneal) nerve. In general we found lateral popliteal nerve action potentials to be smaller than those seen in the upper limb but, once initial technical difficulties had

*Clinical research fellow, M.R.C. been overcome, the method proved to be of considerable practical value in the assessment of patients with neurological disorders affecting the lower limbs.

\section{Material and Methods}

Observations were made on 39 control subjects. Thirty-five patients with spinal cord or peripheral nerve lesions affecting the legs were also studied.

Subjects were examined lying supine on a couch in a warm room; to minimize cooling they were covered with blankets except for the leg and foot under examination which were loosely wrapped in cotton wool. Complete muscular relaxation of the leg during the recording was best obtained by placing a small pillow or rolled blanket under the thigh so that the knee was slightly flexed; in some cases a slight degree of external rotation at the hip was also helpful. A stimulating cathode was placed over the anterior tibial nerve on the dorsum of the ankle, its position being such that stimulation produced brisk contractions of the extensor digitorum brevis and paraesthesiae radiating into the first interdigital cleft. In some patients with peripheral nerve lesions the normal motor and sensory response to nerve stimulation could not be obtained, and the stimulating cathode was then placed over the expected course of the nerve at the junction of the outer and middle thirds of a line joining the medial and lateral malleoli. A circular pad electrode with a diameter of $1.5 \mathrm{~cm}$. was used as the stimulating cathode. In most experiments the anode was a metal plate $3.5 \mathrm{~cm}$. $\times 6 \mathrm{~cm}$. strapped to the outer border of the sole, but when simultaneous recording from the extensor digitorum brevis was necessary, the anode was placed behind the lateral malleolus in order to reduce stimulus artefact. These anode positions were chosen for convenience and did not affect nerve conduction times. In each case the stimulus was a condenser discharge with a time constant of $150 \mu \mathrm{sec}$. delivered through an isolating transformer (ratio $1: 1$ ), the peak voltage being continuously variable up to $300 \mathrm{v}$. In a few experiments, to be described in detail in a later section, two stimuli of independently variable intensity and delay were used.

For recording from the lateral popliteal nerve at the neck of the fibula, both surface and needle electrodes were tried; their relative advantages will be described in 
a later section. The action potential of the afferent volley was amplified and displayed on one beam of a doublebeam oscilloscope. The second beam usually displayed a time-scale, but on a few occasions it was used for a simultaneous display of muscle action potentials from the extensor digitorum brevis. A differential amplifier was used with a time constant of approximately $50 \mathrm{msec}$. and a high frequency response which was not reduced by more than $3 \mathrm{db}$ at $5 \mathrm{kc} / \mathrm{s}$. In a few experiments of the type illustrated in Fig. 5 the time constant was reduced to $15 \mathrm{msec}$. and the h.f. response heavily cut above $1 \mathrm{kc} / \mathrm{s}$. The effect of these changes on a rectangular pulse is shown in Fig. $5 f$.

A stimulus repetition rate of $1 / \mathrm{sec}$. was found to be convenient and for photographic recording of the nerve action potential 100 successive sweeps at low brilliance were superimposed. In some experiments a single trigger pulse was used to initiate the sweep, the stimulus, and a millisecond time scale. With this arrangement the onset of the stimulus artefact was not recorded, but latency could be measured directly from the beginning of the time scale which was always visible however large the stimulus artefact. In other experiments a delay circuit made it possible to display the whole stimulus artefact, and in such cases latency was measured from the beginning of the artefact.

\section{Results}

Choice of Recording Electrodes.-With a stimulating cathode over the anterior tibial nerve on the dorsum of the ankle, we first attempted to record the action potential of the afferent volley from the lateral popliteal nerve at the neck of the fibula (Fig. 1) using the surface recording electrodes originally described by Dawson and Scott (1949). These electrodes had proved satisfactory in the upper limb but it soon became clear that they were unlikely to be adequate for our purpose; lateral popliteal nerve action potentials were found to be very much smaller than those recorded previously in the arm, and in some normal subjects their amplitude was close to the noise level of the amplifier. Particular difficulty was experienced in patients with obese or oedematous legs, and in order to decrease the distance between the recording electrodes and the nerve trunk, surface electrodes were replaced by two small stainless steel needles (No. 18, hypodermic) placed at right angles to the course of the nerve and inserted to a depth of $1.5 \mathrm{~cm}$. No attempt was made to pierce the nerve sheath, the lower needle being immediately behind the neck of the fibula and the upper needle behind the tendon of the biceps femoris at its insertion. With this arrangement each electrode lay just behind the nerve trunk, the interelectrode distance varying between 3 and $4 \mathrm{~cm}$. in different experiments.

Direct comparison between surface and needle recording was made in eight control subjects, the

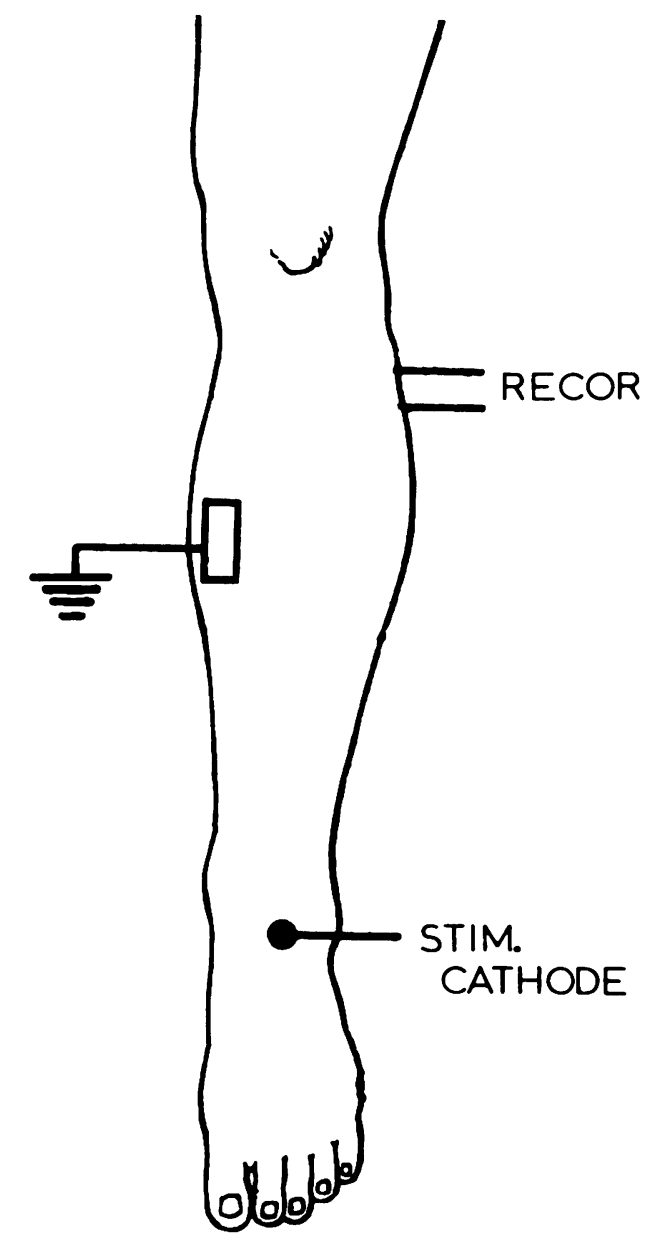

FIG. 1.-Position of stimulating and recording electrodes.

inter-electrode distance being similar for the two types of electrode. With buried needles the amplitude of the recorded potential was always greater than that obtained with surface electrodes; the increase in amplitude in different subjects ranged from $11 \%$ to $93 \%$, the mean increase for the eight subjects being $42 \%$. A typical result is shown in Fig. 2, trace a (needles) and trace b (surface electrodes) having been made successively without any change in the stimulus applied to the anterior tibial nerve at the ankle. It can be seen that in each case a $\delta$ triphasic action potential was recorded, the main upward deflection of the trace indicating negativity of the lower electrode with respect to the upper one. $>$ For the initial upward deflection the amplitude with needle recording (a) was $7.6 \mu \mathrm{v}$ as compared with $5.4 \mu \mathrm{v}$ for the surface recording (b). However, when peak-to-peak amplitude was measured in order to 

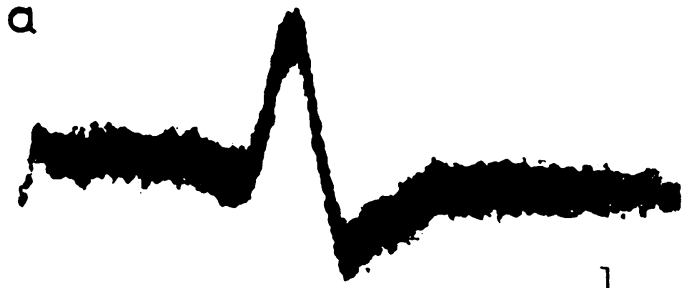

b

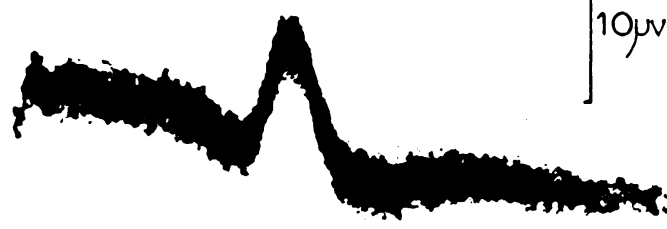

msec

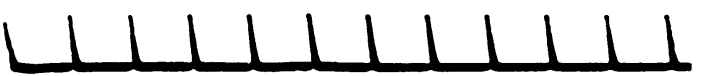

Fig. 2.-Control subject (M.A.). Action potentials recorded from the lateral popliteal nerve using (a) needle and (b) surface electrodes. Stimulus to anterior tibial nerve at the ankle unchanged throughout. One hundred faint traces superimposed.
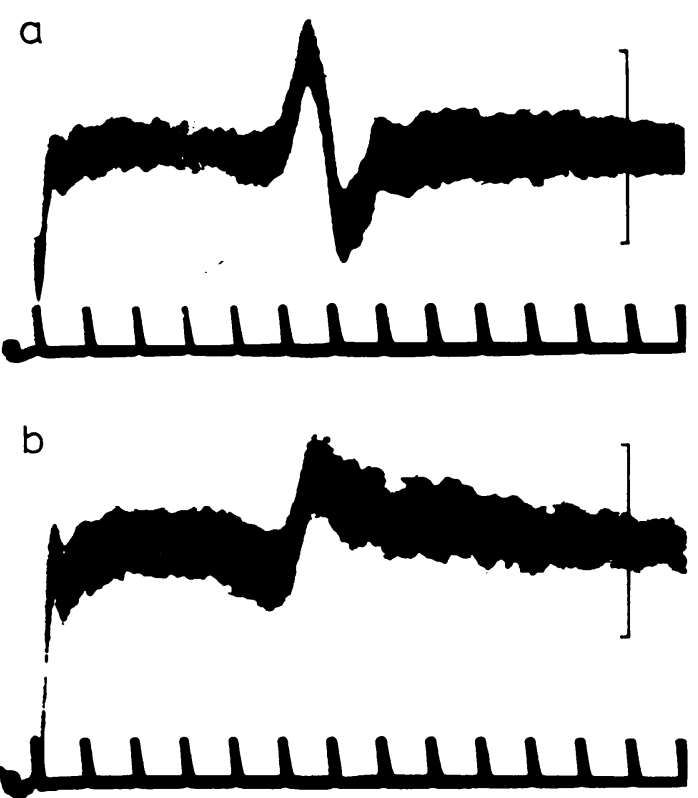

include the prominent late deflection in the needle record the difference in amplitude was much greater $(11.5 \mu \mathrm{v}$ compared with $6.7 \mu \mathrm{v})$. From this and similar experiments it was clear that a substantial increase in the amplitude of the recorded potential could be obtained by the insertion of needle electrodes. For this reason the use of surface electrodes was abandoned and further attention concentrated on the use of implanted needles.

Experiments were then carried out on eight subjects to determine the contribution of each electrode to the recorded potential. For this purpose recordings were first made through a pair of needles at the head of the fibula, after which further recordings were made between each needle of the pair and an indifferent electrode $20 \mathrm{~cm}$. higher on the lateral aspect of the thigh; the stimulus to the anterior tibial nerve on the dorsum of the ankle remained unchanged throughout. Fig. 3a shows the typical triphasic action potential recorded from a pair of fibular needles, the tip of the lower needle being behind the neck of the fibula and the upper needle tip behind the tendon of the biceps femoris at its insertion. As before, an upward deflection of the trace indicates negativity of the lower electrode with respect to the upper one. In Fig. 3b is shown the action potential recorded between the lower needle of the pair and the indifferent electrode, and in Fig. 3c the action potential recorded between the upper of the pair and the indifferent electrode; in both cases the polarity was such that an upward deflection of the trace indicates negativity of the fibular electrode with respect to the indifferent one. From Fig. 3 it is clear that each fibular electrode became active in turn, the time for propagation of the action potential over the $3.0 \mathrm{~cm}$. of nerve between the two electrodes being sufficient to allow

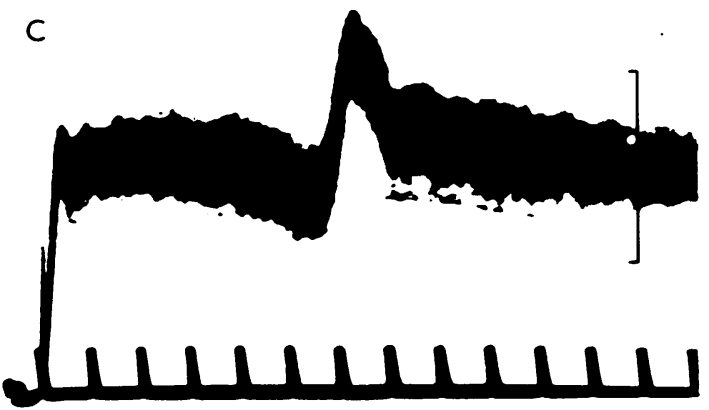

Fig. 3.-Control subject (M.D.). Action potentials from the lateral popliteal nerve; stimulus to anterior tibial nerve at the ankle unchanged throughout.

a. Bipolar recording from two needles $3 \mathrm{~cm}$. apart along the course of the nerve at the head of the fibula.

b. Monopolar recording from lower fibular electrode and distant reference electrode.

c. Monopolar recording from upper fibular electrode and distant reference electrode.

Calibration $10 \mu \mathrm{v}$; time scale in msec.; 100 faint traces super imposed. 


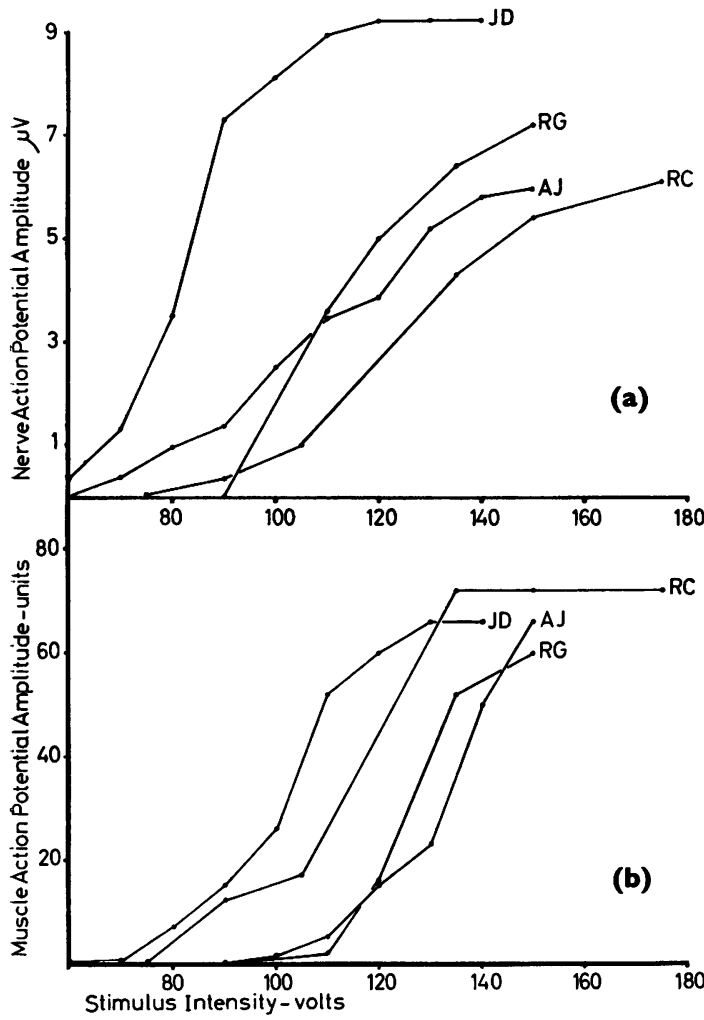

FIG. 4.-Growth of nerve action potentials from lateral popliteal nerve (a) and of muscle action potentials from the extensor digitorum brevis, (b) with increasing intensity of stimulus to anterior tibial nerve at ankle, to show relative excitability of motor and sensory fibres. Curves identified by initials of subjects.

both phases to appear in the bipolar record (Fig. 3a) without significant attenuation.

In the light of this information one can interpret the difference between the surface and needle records shown previously in Fig. 2. The absence of a large third (downward) deflection in the surface record (Fig. 2b) suggests that the upper electrode of the pair was too far from the nerve trunk to make a substantial contribution to the recorded action potential. The needle record (Fig. 2a), on the other hand, shows a prominent third (downward) deflection suggesting that the tip of the upper needle was relatively close to the lateral popliteal nerve as it passed upwards through the lateral part of the popliteal fossa.

From the experiments described above it was concluded that paired needles were probably the most satisfactory electrodes for routine recording. It might be argued that the use of a single exploring electrode at the neck of the fibula with an indifferent electrode on the thigh should be prefered to bipolar recording owing to the simplicity of the recorded $Z$ waveform in the former case. However, with $a)$ single active electrode and an indifferent electrode, much more random muscle activity was recorded than with paired fibular electrodes placed sym- 0 metrically and relatively close together. For this reason monopolar records were often spoilt by muscle activity whereas a stable baseline could still $\underset{F}{\stackrel{0}{F}}$ be obtained by using paired fibular electrodes in the same patient.

Relative Excitability of Motor and Sensory Fibres.-The effect of varying stimulus intensity was studied in four control subjects. The stimulus was? applied to the anterior tibial nerve on the dorsum of $\mathcal{\mathbb { Q }}$ the ankle, and the nerve action potential recorded $\%$ through paired electrodes at the head of the fibula. $\vec{\circ}$ In each case the muscle action potential was recorded $\rightarrow$ simultaneously from the extensor digitorum brevis $\vec{\omega}$ through surface electrodes, and displayed on the second beam of the oscilloscope. Results from the four subjects are shown in Fig. 4. Nerve action potential amplitude is shown in Fig. 4a and muscle + action potential amplitude in $4 \mathrm{~b}$; both are plotted against the stimulus intensity in volts. Individuglo curves are identified by the initials of the subjects. It will be seen that in subjects J.D., R.G., and A nerve action potentials were present at a stimul@s $Z$ intensity insufficient to excite motor fibres, whereas? in subject R.C. the nerve and muscle action potentiags appeared together. With increasing stimulus strenghto a point was reached in subject J.D. beyond which the amplitude of the nerve action potential remain $\overrightarrow{\text { de }} \overrightarrow{0}$ constant. In R.C., R.G., and A.J. nerve action?potential amplitude continued to grow throughout the range of stimulus intensity tolerated by the subject, although the increase in size was relatively small at the upper end of the range.

It may be noted that in these experiments threeळ of the four subjects were unable to tolerate maximal stimuli. This was probably due to the fact that eacho응 recording involved the superimposition of 100 faint $^{3}$ traces so that a full stimulus-response curve was derived from 1,000 or more stimuli. In these circumstances, the repeated use of a stimulus which was? even mildly painful was sufficient to prevent the complete muscular relaxation necessary for record- $-\frac{0}{3}$ ing nerve action potentials of small amplitude. However, it is clear from these experiments that in normal subjects the intensity of a stimulus to the anterior tibial nerve can be such that it will excite? most of the large fibres in the nerve.

It was mentioned above that in three of the four subjects a nerve action potential could be recordedr with a stimulus at the ankle too weak to excite motor fibres in the same nerve trunk, and fromo 


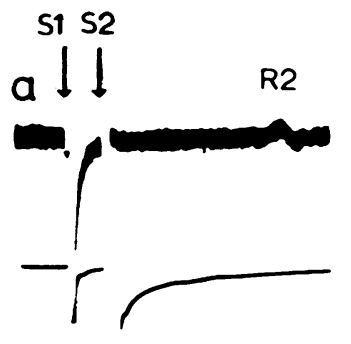

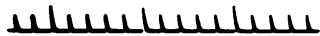

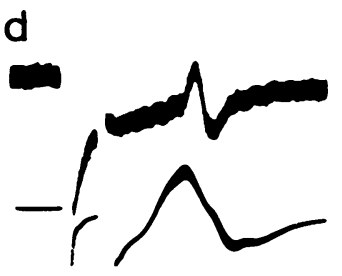

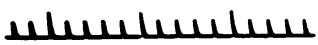

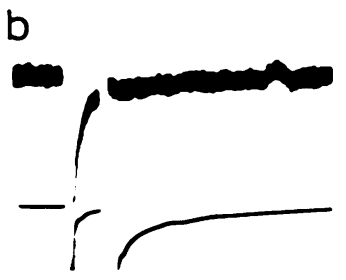

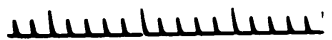

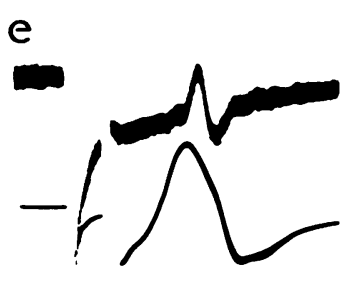

Mhumentures
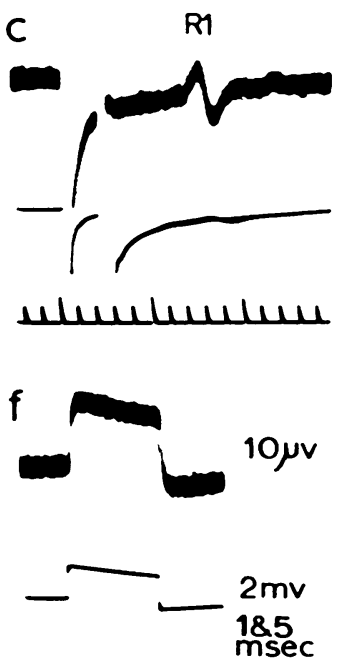

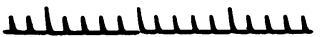

FIG. 5.-Subject R.G. Upper trace shows nerve action potentials recorded through electrodes 7 and 8. Lower trace shows muscle potentials from the extensor digitorum brevis recorded through electrodes 3 and 4. Stimulus $\mathbf{S 2}$, producing small potential $\mathbf{R 2}$ in upper trace, was unchanged throughout experiment. Stimulus S1, preceding S2 by 2 msec., was gradually increased from subthreshold in a to maximal in e. Nerve action potential $R 1$ appears in c, with diminution of $\mathbf{R 2}$, at a time when stimulus $\mathrm{S} 1$ at threshold for motor fibres.

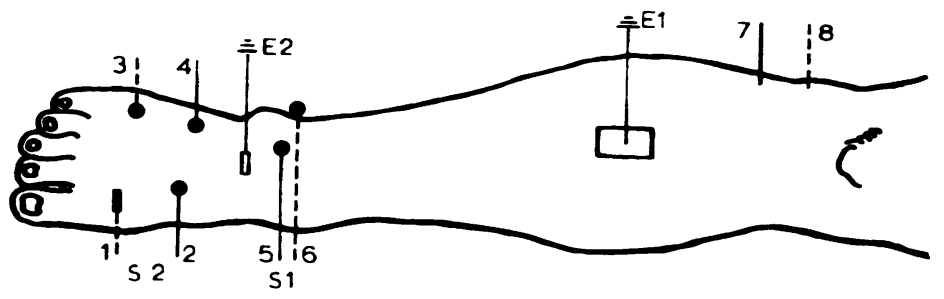

Fig. 4 it can be seen that for subjects J.D., A.J., and R.G. the nerve action potential had already reached $11 \%, 38 \%$, and $50 \%$ of maximum amplitude by the time the first motor response was seen. A similar difference in the thresholds of motor and sensory fibres was described by Dawson (1956) in the median and ulnar nerves at the wrist; it was suggested by Dawson that some at least of these low-threshold afferent fibres came not from the muscles but from the fingers. In order to investigate the origin of lowthreshold afferent fibres in the anterior tibial nerve, an experiment originally devised by Dawson was adapted for use in the foot. The experimental arrangement is shown under Fig. 5. The anterior tibial nerve was stimulated at the ankle through electrodes 5 and 6 , the lateral popliteal nerve action potential and the muscle action potential from the extensor digitorum brevis being recorded simultaneously through electrodes 7 and 8 and 3 and 4 respectively. A further pair of stimulating electrodes 1 and 2 was placed over the cutaneous branch of the anterior tibial nerve on the dorsum of the foot, the distance between the two stimulating cathodes (electrodes 2 and 5) being
$10.5 \mathrm{~cm}$. The stimulus $\mathrm{S} 2$ was set to follow $\mathrm{S} 1$ by an interval of $2 \mathrm{msec}$, the two stimuli being of identical waveform and duration but of independently variable intensity. The strength of $\mathbf{S} 2$ was sufficient to excite the sensory fibres of the anterior tibial nerve and it gave rise to a small action potential R2 recorded through electrodes 7 and 8. This stimulus was kept constant throughout the experiment. The stimulus S1, preceding S2 by 2 msec., was initially too weak to excite either motor or sensory fibres at the ankle but was gradually increased to near maximal during the experiment. The results of one such experiment are shown in Fig. 5 a to e, the nerve action potentials being displayed on the upper trace and the muscle action potentials below. In Fig. 5a, S1 was subthreshold and the small nerve action potential (R2) due to $\mathrm{S} 2$ is seen with a latency of $9.8 \mathrm{msec}$. to peak. In Fig. $5 \mathrm{~b}, \mathrm{~S} 1$ was sufficient to cause slight irregularity of the upper trace which may be presumed to represent the beginning of a nerve action potential from S1. With a further increase in the strength of $S 1$ this action potential (R1) is clearly seen in c; this record also 
shows the first trace of a muscle response. The strength of $\mathrm{S} 1$ was further increased in d and again in e. No further change in either nerve or muscle action potential amplitude resulted from increasing $\mathrm{S} 1$ beyond this intensity. It will be seen that in c the S1 shock was close to motor threshold so that the nerve action potential R1, whose amplitude was already $50 \%$ of maximum, may be assumed to be derived almost entirely from sensory fibres. The question as to whether these fibres are muscle or cutaneous afferents is answered by the effect of S1 on the S2 volley from the dorsum of the foot. The small action potential $\mathrm{R} 2$ produced by this volley is clearly seen in Fig. 5a and b, but is largely abolished by $\mathrm{S} 1$ in $\mathrm{c}$, indicating that the same fibres were being stimulated at a higher level. Complete blocking of the S2 volley due to a further increase in $\mathrm{S} 1$ intensity can be seen in $d$ and $e$. From this experiment it appears that the cutaneous branch of the anterior tibial nerve can be excited by a stimulus insufficient to excite motor fibres. This does not imply that there is necessarily a true difference in the electrical threshold of the two sets of fibres; it could merely mean that the cutaneous branch is more superficially placed and therefore more easily excited. However, the result is of practical importance in that it makes it possible to excite sensory fibres in isolation from motor fibres, so that conduction velocity in the two groups can be compared.

Relative Conduction Velocities of Motor and Sensory Fibres.-In three of the four subjects described in the previous section, sensory fibres in the anterior tibial nerve at the ankle were stimulated by a weak shock which was insufficient to excite motor fibres; in each case a small sensory action potential was recorded at the head of the fibula using surface electrodes. The intensity of the stimulus at the ankle was then increased until it was maximal for the motor fibres to the extensor digitorum brevis and motor latency was recorded through surface electrodes in the position shown for the pair 3 and 4 in Fig. 5. Finally, the motor fibres to the extensor digitorum brevis were stimulated at the head of the fibula through the surface electrodes used previously for recording the nerve action potenti l (in the position shown for electrodes 7 and 8 in Fig. 5), the stimulus again being maximal for motor fibres.

Results in the three subjects are shown in Table $I$. Latencies to the base and to the peak of the sensory action potential are given separately in the first two columns of the table. Motor latencies with stimulation at the ankle and at the head of the fibula are shown in the third and fourth columns of Table I and motor conduction time between the two stimulus
TABLE I

MOTOR AND SENSORY CONDUCTION IN THREE HEALTHY SUBJECTS

\begin{tabular}{|c|c|c|c|c|c|}
\hline \multirow{2}{*}{ Subject } & \multicolumn{2}{|c|}{$\begin{array}{c}\text { Sensory Latency } \\
\text { (msec.) }\end{array}$} & \multicolumn{2}{|c|}{$\begin{array}{c}\text { Motor Latency } \\
\text { (msec.) }\end{array}$} & \multirow{2}{*}{$\begin{array}{c}\text { Motor } \\
\text { Conduction } \\
\text { Time (msec.) } \\
\text { Knee to } \\
\text { Ankle }\end{array}$} \\
\hline & $\begin{array}{c}\text { To } \\
\text { Base }\end{array}$ & $\begin{array}{c}\text { To } \\
\text { Peak }\end{array}$ & $\begin{array}{l}\text { From } \\
\text { Ankle }\end{array}$ & $\begin{array}{c}\text { From } \\
\text { Knee }\end{array}$ & \\
\hline $\begin{array}{l}\text { J.D. } \\
\text { R.G. } \\
\text { A.J. }\end{array}$ & $\begin{array}{l}5 \cdot 8 \\
7 \cdot 2 \\
6 \cdot 6\end{array}$ & $\begin{array}{l}6.4 \\
7 \cdot 7 \\
7.2\end{array}$ & $\begin{array}{l}3.8 \\
3.8 \\
3.8\end{array}$ & $\begin{array}{l}10.1 \\
11.1 \\
10.8\end{array}$ & $\begin{array}{l}6 \cdot 3 \\
7 \cdot 3 \\
7 \cdot 0\end{array}$ \\
\hline
\end{tabular}

positions is shown in the last column. In this way a direct comparison may be made between conduction times in motor and sensory fibres in the same nerve trunk, measured in both cases over the same distance between the knee and ankle. From Table $I$ i it can be seen that in all three subjects conduction $\vec{\circ}$ time for the fastest sensory fibres (i.e., latency measured to the base of the nerve action potential) was slightly shorter than motor conduction time over the same distance.

In the case of sensory fibres, conduction time includes excitation time at the site of nerve stimulation, which is of the order of $0.1 \mathrm{msec}$. (Hunt, 1954) Motor conduction time is obtained by subtractiog o of one latency from another and no correction for excitation time need be made; thus the absolute differences in conduction time between motor and $z$ sensory fibres are probably slightly greater that those shown. It may be concluded that in thes subjects, conduction velocity in the fastest sensor fibres of the anterior tibial nerve was slightly greater than in the fastest motor fibres to the extensor digitorum brevis.

The Range of Normal Variation.-In order to establish the range of normal variation, 39 control subjects were examined. Observations were made on a few healthy subjects working in the laboratory $\mathbb{D}$ and also on patients of widely varying ages, all of whom were without evidence of local pathology in the leg. The clinical diagnosis in control subjects is shown in Table IJ from which it will be seen that

TABLE II

CLINICAL DIAGNOSIS IN 39 CONTROL SUBJECTS

\begin{tabular}{ll|lr}
\hline Epilepsy & 9 & Carpal tunnel syndrome & 2 \\
Lateral popliteal nerve palsy & 4 & Cervical syringomyelia & 1 \\
Cerebral tumour & 4 & Gallstones & 1 \\
Cerebral vascular disease & 2 & Hysteria & 1 \\
Dementia & 2 & Anterior tibial nerve lesion & 1 \\
Migraine & 3 & Parkinsonism & 1 \\
Cervical spondylosis & 2 & Healthy subjects & 4 \\
Ulnar neuritis & 2 & Total & 39 \\
\hline
\end{tabular}

four patients with lateral popliteal palsies and one with a suspected anterior tibial nerve lesion were 
included; in these cases control measurements were made on the unaffected leg.

In each case the anterior tibial nerve was stimulated on the dorsum of the ankle and the action potential of the afferent volley in the lateral popliteal nerve recorded through a pair of No. 18 hypodermic needles. The lower needle was always inserted at right angles to the skin at the level of the neck of the fibula so that its tip lay $1.5 \mathrm{~cm}$. from the surface immediately behind the bone. The upper needle was placed parallel to it and 3 to $4 \mathrm{~cm}$. higher in the leg, its tip lying behind the tendon of biceps femoris close to its insertion into the head of the fibula.

Action potentials were recorded successfully in all 39 control subjects. In each case the growth of the nerve action potential with increasing stimulus strength was watched on the oscilloscope until a point was reached at which its amplitude appeared to remain constant. Photographic records were then made. Peak-to-peak amplitude of the potentials is shown in Fig. 6, plotted against the subject's age in years, and it can be seen that values ranged from 2 to $15.5 \mu \mathrm{v}$. While there appears to be some diminution in the size of the potentials in the older age groups, the scatter of the results in younger subjects is such that the difference with age is not statistically significant.

For the estimation of conduction time, latencies were measured both to the base and to the peak of the main deflection of the action potential, this deflection being due to negativity of the lower recording electrode with respect to the upper one. Although the time of arrival of impulses at the lower recording electrode is indicated by the onset (i.e., the base) of the negative deflection, measurement to this point was difficult in the case of small potentials, whereas latencies to peak could be measured accurately. In the 39 control subjects peak latencies ranged from $5.3 \mathrm{msec}$. to $8.8 \mathrm{msec}$. and in spite of scatter showed an approximately linear relation to conduction distance.

When conduction distance is divided by latency an estimate of conduction velocity is obtained. Velocity calculated in this way inevitably includes certain errors; on the one hand the excitation time of the nerve will tend to reduce it, whereas any spread of current from under the stimulating cathode will tend to increase it. However, in relation to the length of nerve examined ( 25 to $35 \mathrm{~cm}$.) these errors are small, and the estimation of velocity does at

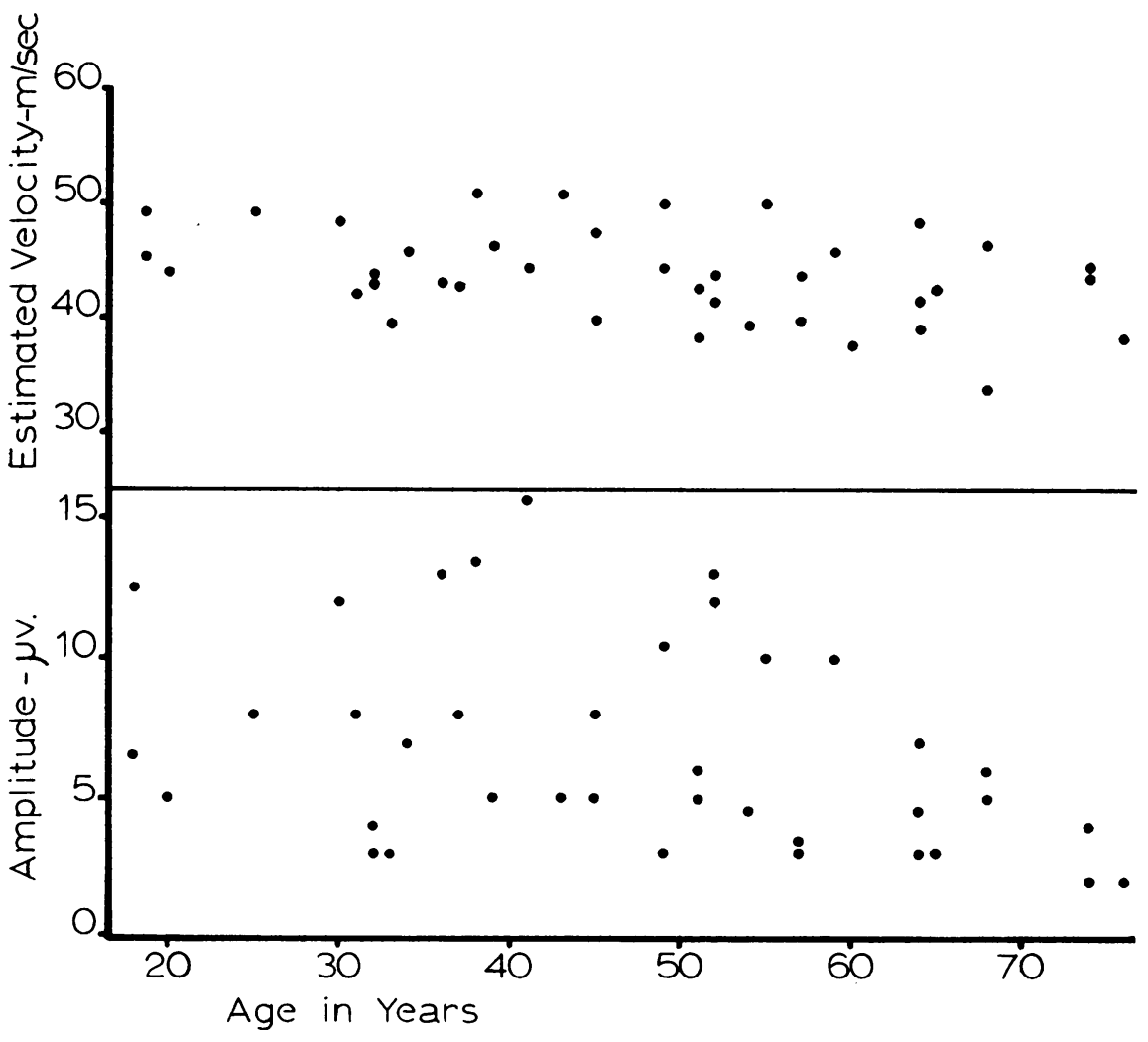

Fig. 6.-Amplitude and conduction velocity of action potentials recorded from lateral popliteal nerve plotted against age in 39 control subjects. 
least provide a correction for conduction distance in different subjects provided that the same technique is used. Conduction velocity derived from peak latency in the 39 control subjects ranged from $33 \cdot 8$ to $51.8 \mathrm{~m} . / \mathrm{sec}$. and in Fig. 6 it has been plotted against the subject's age in years. Some decrease in conduction velocity in the older age groups is apparent; there were, for example, 19 subjects over the age of 50 and 20 subjects under this age; mean velocity was $42.6 \mathrm{~m}$./sec. (S.D. \pm 4.6 ) in the former, and $45.5 \mathrm{~m}$./ sec. (S.D. \pm 3.5 ) in the latter group, this difference being statistically significant $(\mathrm{p}<0.05)$.

It has already been pointed out that latency measured to the base of the action potential is unreliable for small action potentials. With potentials of more than $5 \mu \mathrm{v}$ in amplitude this difficulty does not arise, and in such cases velocities derived from base and peak measurements may be compared. There were 19 subjects in the control group with nerve action potentials greater than $5 \mu \mathrm{v}$ in amplitude and their results are shown graphically in Fig. 7. Base velocity in the 19 subjects ranged from $47 \cdot 3$ $\mathrm{m} . / \mathrm{sec}$. to $63.0 \mathrm{~m}$. $/ \mathrm{sec}$. (mean $53.0 \mathrm{~m}$. $/ \mathrm{sec}$., S.D. \pm 3.85 ) whereas peak velocity ranged from $39.8 \mathrm{~m}$. $/ \mathrm{sec}$.

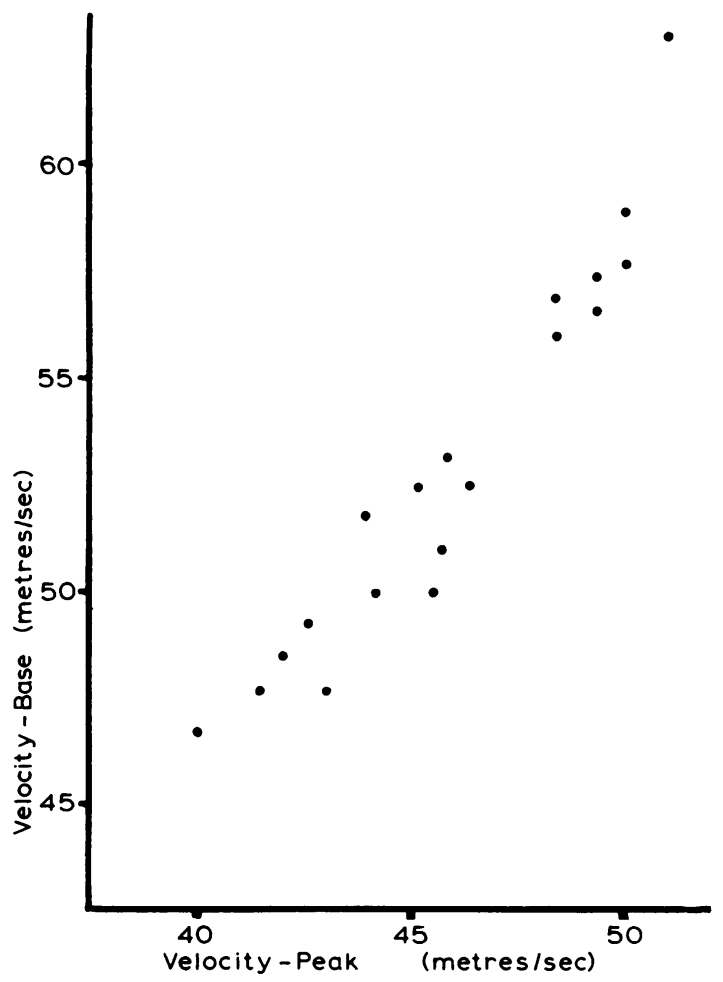

Fig. 7.-Conduction velocity calculated from latency to base of action potential plotted against velocity calculated from peak latency in 19 control subjects. to $51.0 \mathrm{~m} . / \mathrm{sec}$. (mean $45.9 \mathrm{~m} . / \mathrm{sec}$. S.D. \pm 3.25 ). These figures may be compared with the range of 35.6 to $63.5 \mathrm{~m}$./sec. (mean $49.7 \mathrm{~m}$./sec., S.D. $\pm 7 \cdot 1$ ) given by Thomas, Sears, and Gilliatt (1959) for the maximal velocity of motor fibres to the extensor 0 digitorum brevis in healthy subjects.

Although a triphasic waveform was seen in most of our control subjects, there were four in whom the nerve action potentials were polyphasic. Polyphasic potentials were also seen in pathological cases and they will be discussed in a later section.

Investigation of Pathological Cases.-Lateral popliteal nerve action potentials were examined in $35 \vec{\pi}$ patients with neurological disorders, the standard technique described for the control subjects being ${ }^{\circ}$ used in each case; the anterior tibial nerve at the $\vec{\circ}$ ankle was stimulated with a maximal or near- $\overrightarrow{\vec{H}}$ maximal shock, and the nerve action potential ${ }^{\circ}$ recorded from the lateral popliteal nerve at the head of the fibula through a pair of No. 18 hypo-z dermic needles placed 3 to $4 \mathrm{~cm}$. apart as previously in described. Since the pathological group contained a proportion of patients with small action potentiats latency was measured to the peak of the ma deflection and values for conduction velocity subsequent sections are derived from peak latenci度 in all cases.

Multiple Sclerosis.-Fourteen patients with mu $\$$ tiple sclerosis were examined. Whereas patiens with spinal cord disease localized to the cervio region (cervical spondylosis, cervical syringomyeliâ. $\vec{\emptyset}$ were included in the control group, those with multiple sclerosis were excluded on the grounds that 0 a plaque in the lumbar cord might affect lateral popliteal nerve action potentials; actual muscle wasting as a result of denervation is known to be rare in this condition but it was felt that lesser $\mathbb{D}$ degrees of interference with the ventral root outflow to the extensor digitorum brevis would be $\frac{0}{3}$ difficult to exclude. While this should not affect sensory impulses it might well modify the antidromic volley in motor fibres and alter its contribution to the action potential.

The ages of the 14 patients with multiple sclerosis ranged from 26 to 51 years, and in Table III the results are compared with those described previously for control subjects of similar age.

It is clear from Table III that the differences

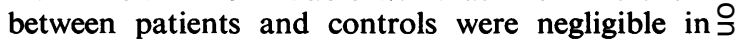
respect of both amplitude and conduction velocity $\rightarrow$ of the nerve action potential. Polyphasic action potentials were seen in three out of 14 patients with $N$ multiple sclerosis, and their significance is discussed in a later section. 
TABLE III

LATERAL POPLITEAL NERVE ACTION POTENTIALS IN MULTIPLE SCLEROSIS

\begin{tabular}{l|c|c|c|c}
\hline & \multicolumn{2}{|c|}{$\begin{array}{c}\text { 14 M.S. Patients } \\
\text { Aged 26-51 }\end{array}$} & $\begin{array}{c}20 \text { Control Subjects } \\
\text { Aged 18-49 }\end{array}$ \\
\cline { 2 - 5 } & Range & Mean & Range & Mean \\
\hline $\begin{array}{c}\text { Conduction velocity } \\
\text { (m./sec.) }\end{array}$ & $38.5-52.8$ & 46.3 & $39.8-51 \cdot 0$ & 45.5 \\
\hline \begin{tabular}{c} 
Amplitude $(\mu v)$ \\
\cline { 2 - 5 }
\end{tabular} & $3-15$ & 7.9 & $3-15 \cdot 5$ & 7.6 \\
\hline
\end{tabular}

Motor Neurone Disease.-Five patients with motor neurone disease were examined; their ages ranged from 54 to 62 years. Patients with severe wasting of the lower limbs were chosen, and in each case the response of the extensor digitorum brevis to nerve stimulation was monitored through surface electrodes placed over the expected position of the muscle, the wasting being of such severity that the muscle belly could no longer be palpated through the skin. In three patients there was no muscle response to stimulation of the anterior tibial nerve at the ankle, and in the other patients only two or three surviving motor units were activated. Although few if any motor nerve fibres were excited by the stimulus, an action potential was recorded in every case from the lateral popliteal nerve at the head of the fibula; action potential amplitude in the five patients ranged from 2.5 to $3.4 \mu \mathrm{v}$ as shown in Table IV.

TABLE IV

NERVE ACTION POTENTIALS IN MOTOR NEURONE DISEASE

\begin{tabular}{l|c|c|c}
\hline Subject & $\begin{array}{c}\text { Age } \\
\text { (years) }\end{array}$ & $\begin{array}{c}\text { Conduction } \\
\text { Velocity (m./sec.) }\end{array}$ & $\begin{array}{c}\text { Amplitude } \\
(\mu \mathrm{v})\end{array}$ \\
\hline D.K. & 62 & $33 \cdot 2$ & $2 \cdot 5$ \\
M.Mc. & 59 & 37.3 & $3 \cdot 3$ \\
M.H. & 54 & 41.5 & 3.0 \\
D.V. & 56 & $40 \cdot 7$ & $3 \cdot 0$ \\
E.W. & 60 & 40.5 & $3 \cdot 4$ \\
\hline
\end{tabular}

From these observations it was concluded that when the motor fibres in the anterior tibial nerve had been virtually eliminated by disease, sensory fibres could still be excited in sufficient numbers to give rise to a recordable action potential.

When the conduction velocities shown in Table IV are compared with those of control subjects over the age of 50 years it is clear that conduction was generally slower in the patients with motor neurone disease. These patients were severely paralysed and their reduced conduction velocities were almost certainly due to the low limb temperature which we were unable to correct by simple measures.

Peripheral Neuritis and Subacute Combined Degeneration of the Spinal Cord.-In contrast to the 2 patients with spinal cord disease, those with peripheral neuritis and subacute combined degeneration showed marked changes in the nerve action potential. Three patients with chronic peripheral neuritis were examined (Table V). In each case muscular weakness

\section{TABLE V}

NERVE ACTION POTENTIALS IN PERIPHERAL NEURITIS, SUBACUTE COMBINED DEGENERATION, HEREDITARY ATAXIA, AND PERONEAL MUSCULAR ATROPHY

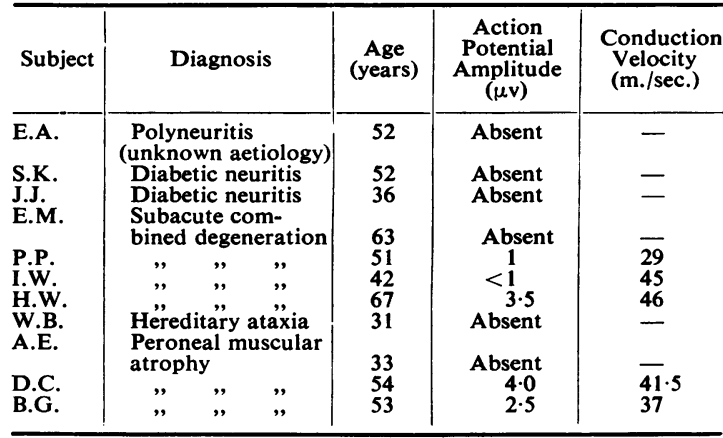

in the legs was accompanied by loss of knee and ankle jerks and impairment of superficial sensation on the feet. In none of these three patients was it possible to record a nerve action potential even when using a stimulus to the anterior tibial nerve of considerably higher voltage than that which would be tolerated by a healthy subject.

It can be seen from Table $V$ that in four patients with subacute combined degeneration the results were more variable; in the case of E.M. the action potential was absent altogether and in P.P. and I.W. minimal deflections of the trace were seen (marked by arrows in Fig. 8 a and $b$ respectively). It may be noted that whereas the minimal deflection shown in Fig. $8 \mathrm{~b}$ had a normal latency, that shown in Fig. 8a was obviously delayed (latency to peak $11.5 \mathrm{msec}$.), from which conduction velocity was estimated to be $29 \mathrm{~m}$./sec. In the fourth patient (H.W.) a triphasic potential of normal latency was recorded (Fig. 8c). It is interesting that in the three patients with much diminished or absent action potentials clinical examination showed mainly a picture of peripheral nerve involvement with flexor plantar responses and superficial sensory loss of stocking distribution. In the fourth patient (H.W.) there was evidence of spinal cord involvement with extensor plantar responses and with preservation of superficial sensation in spite of gross impairment of joint sense.

Hereditary Ataxia and Peroneal Muscular Atrophy.-In one patient with hereditary ataxia (W.B.), a pyramidal and cerebellar syndrome was accompanied by pes cavus, scoliosis, and cardiac 

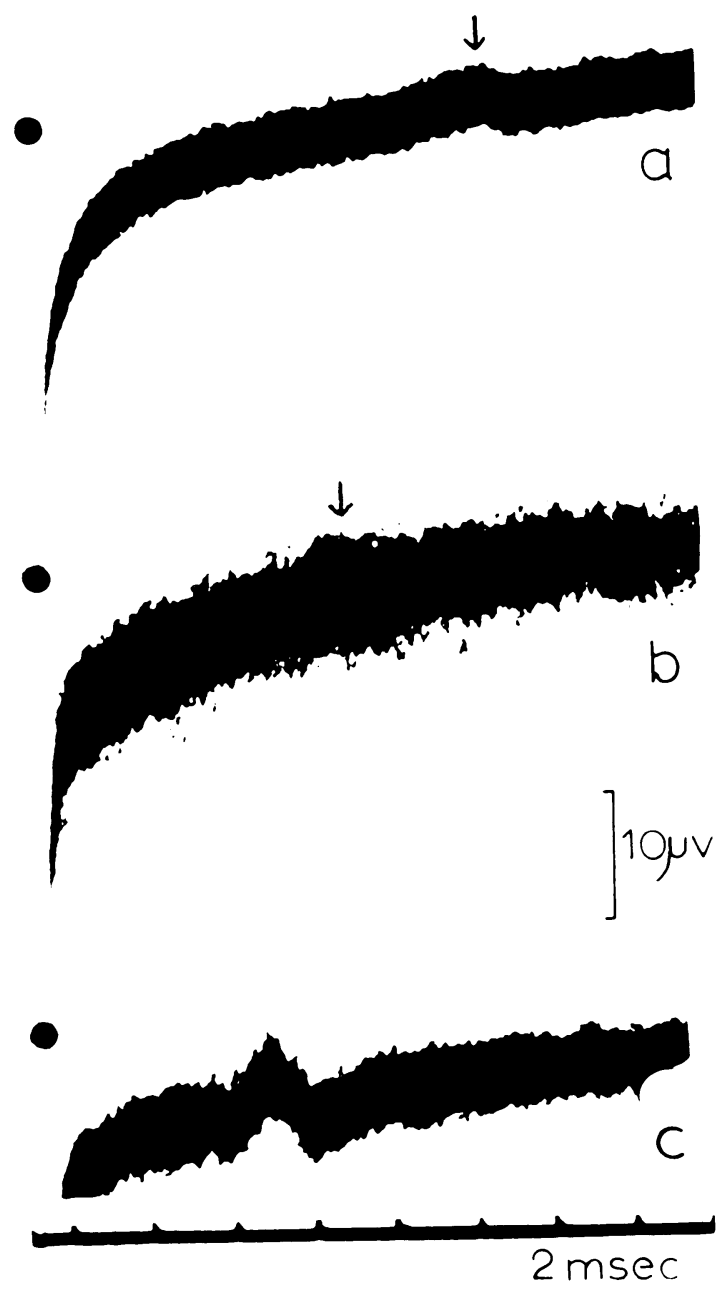

FIG. 8.-Action potentials recorded from the lateral popliteal nerve in three patients with subacute combined degeneration. Stimulus at mark. One hundred faint traces superimposed.

changes. In this patient some wasting of the leg muscles was present with impairment of both deep and superficial sensation in the feet, and it can be seen from Table $\mathrm{V}$ that the lateral popliteal nerve action potential was absent.

Three patients with classical peroneal muscular atrophy (Charcot-Marie-Tooth disease) were examined. In A.E. the only sensory abnormality on clinical testing was some loss of vibration sense in the feet but the lateral popliteal nerve action potential was absent. In D.C. superficial sensation was preserved but there was mild impairment of both vibration and joint sensation in the feet. In B.G. appreciation of vibration and joint sensation was intact, the only clinical sensory abnormality being mild subjective blunting of pin-prick on the toes $\overline{\bar{Z}}$ compared with the shins. In both D.C. and B.G. low-voltage polyphasic potentials were recorded.

Lateral Popliteal (Peroneal) Nerve Palsy.-Four patients with unilateral foot-drop of acute onset were seen. One patient first noticed his symptoms on recovering from a general anaesthetic after an abdominal operation; a second patient developed foot-drop after a particularly vigorous game of football. In the other two cases there was no $\stackrel{9}{\rightarrow}$ definite history of trauma, but in each of them the onset was abrupt, the patient suddenly becoming aware of weakness of the ankle and numbness of the dorsum of the foot. The patients were examined $\stackrel{\mathbb{Q}}{\varrho}$ between four and 12 weeks after the onset, by which time all reported improvement compared with their $\vec{\circ}$ initial condition. No wasting of the anterior tibial or peroneal muscles was noted but weakness was still present in every case. There were mild sensory changes over the dorsum of the foot and toes in all four patients, subjective diminution to pin-prick and cotton wool being present without complete loss of either modality. In two patients the peroneal nerve on the affected side was considered to be thickenef in the region of the neck of the fibula. The nervor system was otherwise normal in every case. $\frac{\rho}{\Phi}$

Results are shown in Table VI, from which it cå Z be seen that there was diminution in action potentir amplitude with slowing of conduction velocity on the affected side in all four patients.

A typical result from one of the four patients shown in Fig. 9. The patient, a clerical worker age 31 , suddenly found when walking to work one morning that his right foot 'flapped' when he put it to the ground; shortly afterwards he noticed an abnormal sensation on touching the skin on the dorsum of the ankle and foot. At the time of the first examination one month after the onset, the $\frac{\mathrm{D}}{\mathrm{Q}}$ patient was already recovering and the only abnormality present on formal neurological examination $\overline{\underline{0}}$ was mild weakness of the anterior tibial and peroneal muscles on the right, with hypalgesia to pin-prick restricted to the dorsum of the foot and toes. When the right extensor digitorum brevis was sampled with a concentric needle electrode no denervation? potentials were seen and the number of motor units under voluntary control was not obviously reduced. With the concentric needle electrode in situ, the anterior tibial nerve was stimulated on the dorsum of the ankle, a muscle action potential of normal 을 latency being recorded (Fig. 9a). When the motor $D$ fibres were stimulated at the neck of the fibula the latency of the muscle response was again normal $\mathrm{N}$ (Fig. 9c), conduction velocity for the fastest fibres $\sigma$ between the two stimulus levels being $48.5 \mathrm{~m} . / \mathrm{sec}$. 
TABLE VI

NERVE ACTION POTENTIALS IN LATERAL POPLITEAL (PERONEAL) PALSY

\begin{tabular}{|c|c|c|c|c|c|}
\hline \multirow[b]{2}{*}{ Subject } & \multirow[b]{2}{*}{ Age } & \multicolumn{2}{|c|}{ Affected Leg } & \multicolumn{2}{|c|}{ Unaffected Leg } \\
\hline & & $\underset{(\mu v)}{\text { Amplitude }}$ & $\begin{array}{c}\text { Conduction Velocity } \\
(\mathrm{m} . / \mathrm{sec} .)\end{array}$ & $\underset{(\mu v)}{\text { Amplitude }}$ & $\begin{array}{c}\text { Conduction Velocity } \\
\text { (m./sec.) }\end{array}$ \\
\hline $\begin{array}{l}\text { C.C. } \\
\text { B.D. } \\
\text { W.P. } \\
\text { G.W. }\end{array}$ & $\begin{array}{l}31 \\
\\
45 \\
68 \\
20\end{array}$ & $\begin{array}{cc}* \mathrm{a} & 3 \cdot 0 \\
\mathrm{~b} & 5 \cdot 0 \\
\mathrm{c} & 6 \cdot 2 \\
& 2 \cdot 0 \\
& 3 \cdot 1 \\
& 3 \cdot 0\end{array}$ & $\begin{array}{ll}\text { a } & 33.2 \\
\text { b } & 38.8 \\
\text { c } & 41.5 \\
& 36.7 \\
& 33.5 \\
& 42.5\end{array}$ & $\begin{array}{l}8.0 \\
\\
8.0 \\
5.8 \\
8.5\end{array}$ & $\begin{array}{l}42 \cdot 0 \\
\\
39 \cdot 8 \\
46 \cdot 3 \\
47 \cdot 5\end{array}$ \\
\hline
\end{tabular}

*Time interval a to $b$, four months; $b$ to $c$, five months.

When this procedure was repeated on the unaffected leg latencies were almost identical (Figs. $9 \mathrm{~b}$ and $9 \mathrm{~d})$, motor conduction velocity being $46 \cdot 3$ $\mathrm{m}$./sec. However, when the anterior tibial nerve was stimulated on the dorsum of the ankle with recording needles in the standard position at the head of the fibula, the amplitude of the nerve action potential on the right was much reduced and could not be increased by raising stimulus intensity. In addition the normal triphasic waveform was lost and latency to peak increased (Fig. 9e). For comparison the

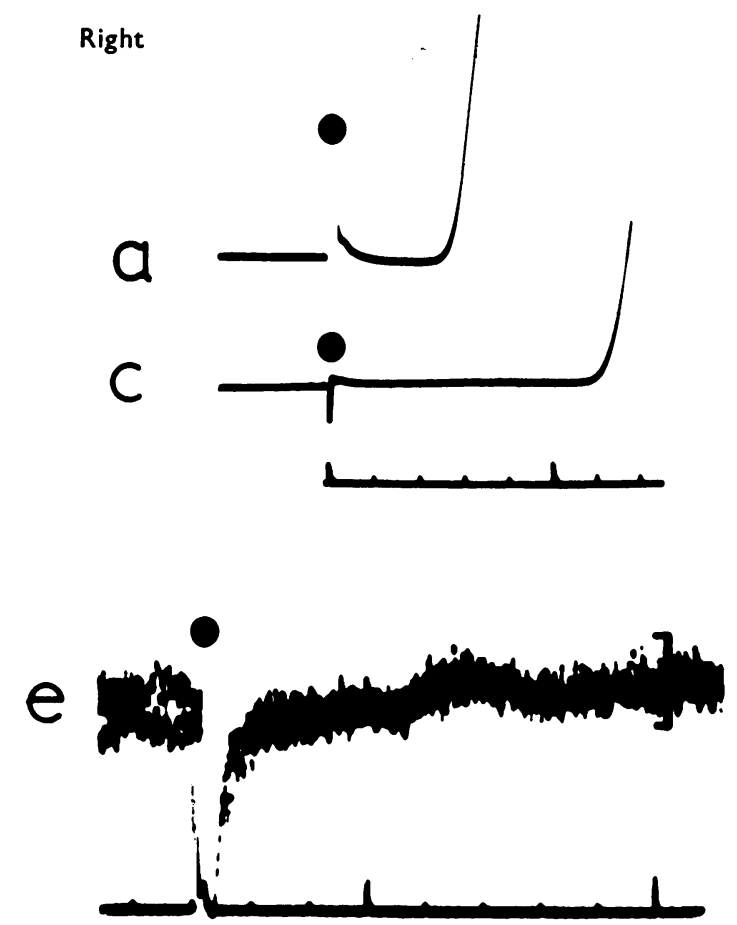

normal nerve action potential recorded from the unaffected leg is shown in Fig. 9f.

A follow-up examination was carried out four months later when the patient appeared to have made a complete clinical recovery. Substantial recovery of the nerve action potential had also occurred (C.C., b, in Table VI) but its amplitude remained less than on the control side. A third examination nine months after the onset showed a further slight increase in the amplitude of the action potential (C.C., c, in Table VI).

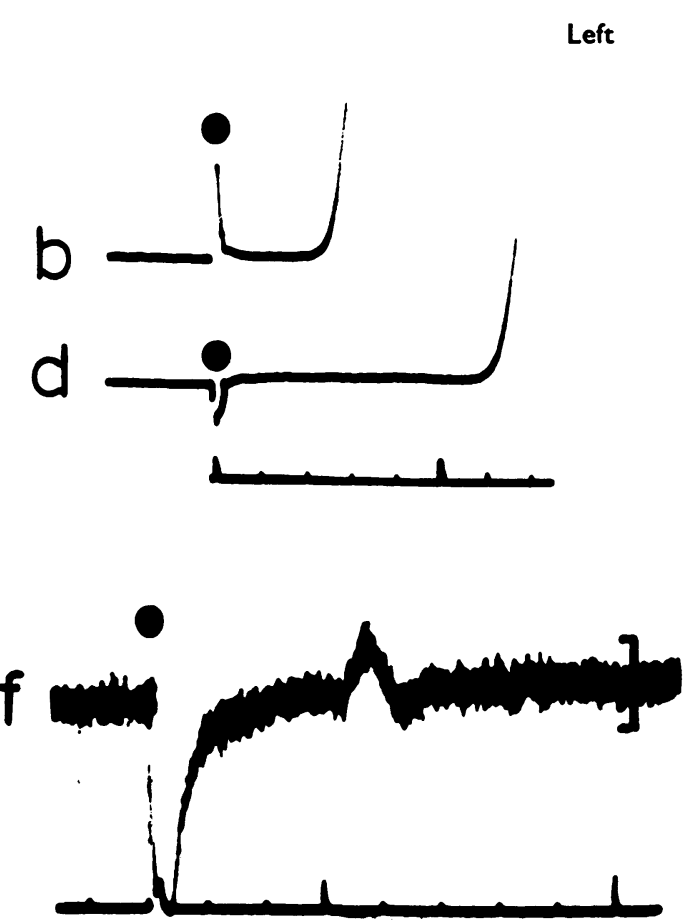

FIG. 9.-Subject C.C. Right lateral popliteal nerve lesion. Onset of muscle action potentials recorded from the extensor digitorum brevis in response to motor nerve stimulation at ankle ( $a$ and $b$ ), and at head of fibula ( $c$ and $d$ ), to show similar motor latencies on the two sides. Nerve action potentials recorded from lateral popliteal nerve at head of fibula with stimulation at ankle (e and $f$ ) show markedly reduced amplitude on affected side. Stimulus at mark and time interval 2 and $10 \mathrm{msec}$. throughout. Five traces superimposed and calibration $10 \mu \mathrm{v}$ for e and f only. 
This case is described in detail to show that with a mild lateral popliteal nerve lesion marked changes in the nerve action potential may occur without any abnormality in motor conduction velocity as estimated from the latencies of the fastest fibres.

Significance of Polyphasic Potentials.-Polyphasic potentials were seen in four control subjects, three patients with multiple sclerosis, and two patients with peroneal muscular atrophy. In each of them the main deflection of the action potential was still recognizable but this was followed by waves of lower amplitude lasting for 2 to $4 \mathrm{msec}$. The investigation of polyphasic potentials was made difficult by the small amplitude of the late components which was less than the amplifier noise level and usually of the order of 0.5 to $1.0 \mu \mathrm{v}$. For this reason departures from normal waveform were not apparent until superimposed records were examined, and some doubt arose as to whether or not the late waves were artefacts arising in the recording system.

In one patient, however, it was possible to investigate the phenomenon further by moving the stimulating cathode proximally along the course of the anterior tibial nerve for a sufficient distance to show that the late waves were propagated with a reduced velocity.

The patient, a taxi-driver aged 36 years, first noticed numbness in the territory of the anterior tibial nerve after falling asleep with his legs on a chair, from which it was suspected that he might have sustained a mild pressure lesion of the anterior tibial nerve at the ankle by sleeping with one foot crossed on top of the other. Except for diminished superficial sensation on the dorsum of the foot and toes, no abnormality could be detected on clinical examination, and the patch of numbness was already fading at the time of the electrical investigation. However, a prolonged polyphasic action potential of low voltage was recorded from the lateral popliteal nerve on the affected side whereas a normal triphasic action potential, $5 \mu \mathrm{v}$ in amplitude, was recorded from the opposite leg. In the records shown in Fig. 10 the nerve action potential was recorded from a single steel needle behind the head of the fibula of the affected leg against an indifferent electrode on the outer surface of the thigh. The stimulus was first applied to the anterior tibial nerve on the dorsum of the ankle slightly below the intermalleolar line (Fig. 10a), after which the stimulating cathode was moved proximally for $5 \mathrm{~cm}$. (Fig. 10b). Comparison of the two traces makes it clear that the late oscillations present are true propagated potentials, latency measurements to early and late components indicating conduction velocities as different as 45 and $26 \mathrm{~m}$./sec. respectively.

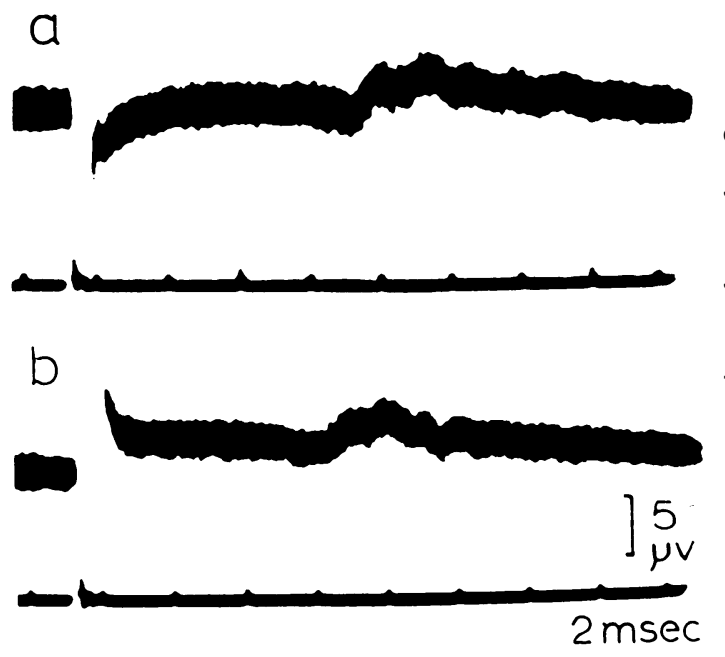

FIG. 10.-Action potentials recorded from the lateral popliteal nerve in a patient (J.B.) with a suspected anterior tibial nerve lesion. Stimulating cathode over anterior tibial nerve was moved $5 \mathrm{~cm}$. proximally between (a) and (b). Recording electrodes unchanged throughout. Records show stimulus artefact followed by low voltage polyphasic action potential in each case. One hundred faint traces superimposed.

With stimulation of the posterior tibial nerve an $\$$ surface recording in the popliteal fossa, Buller (1959\% was able to demonstrate differential velocities of early and late components of the medial popliteal nerv8 action potential in healthy subjects. His results together with our own findings in the patient cites above, suggest that the late components recorded i our other cases may also have been due to activity in slowly conducting fibres. However, their investigation clearly requires apparatus with a higher signal-to-noise ratio than that used in the present work. This should prove to be a problem of great interest when suitable recording apparatus becomes available.

\section{Discussion}

In the upper limbs it is possible to stimulate the fingers and record an action potential from the median or ulnar nerves at the wrist (Dawson, 1956). Alternatively it is possible to stimulate at the wrist and record nerve action potentials at a higher level in the arm (Dawson and Scott, 1949). In patients with neurological disorders, these techniques have already proved to be of considerable practical value, and the present investigation is an attempt to extend음 them to the nerves of the lower limbs. We have not $D$ succeeded in recording action potentials from the anterior or posterior tibial nerves at the ankle with stimulation of the digital nerves in the toes, and have concentrated upon recording from the lateral 
popliteal nerve at the head of the fibula with stimulation of the anterior tibial nerve at the ankle. At both levels the nerve fibres are superficial and are easily located through the skin so that stimulating and recording electrodes can be accurately placed. In this respect the lateral popliteal is a more convenient nerve to examine than the medial popliteal which lies deeply in the popliteal fossa, and we have not attempted to record from the latter. However, Buller (1959) has already done this successfully in healthy subjects using a storage tube, and this presents a promising field for further investigation.

In spite of the superficial position of the lateral popliteal nerve, it became clear at the start of the present work that the action potentials we were attempting to record were very much smaller than those recorded previously from the median and ulnar nerves in the arm. Even when surface electrodes were replaced by needles inserted close to the lateral popliteal nerve, the potentials did not exceed $15 \mu \mathrm{v}$ in amplitude, which may be compared with the range of 33 to $117 \mu \mathrm{v}$ reported for the ulnar nerve in the forearm by Gilliatt and Thomas (1960). However, the technical difficulties of recording small potentials need not be exaggerated. Provided that muscular relaxation is adequate, potentials with an amplitude substantially less than the amplifier noise level can be recorded successfully by photographic superimposition of a number of faint traces, and in our 39 control subjects there was no instance in which we failed to record a potential from the lateral popliteal nerve.

The anterior tibial nerve at the ankle contains motor fibres to the extensor digitorum brevis as well as sensory fibres from the dorsum of the foot, and a stimulus in this situation may thus be expected to excite both groups of fibres. In attempting to analyse the extent to which impulses in sensory fibres contribute to the mixed nerve action potential, we have been helped by the fact that patients with motor neurone disease commonly show complete loss of motor fibres to the extensor digitorum brevis with preservation of sensation. From our observations on these patients it is clear that sensory fibres are present in the anterior tibial nerve in sufficient numbers to produce a recordable action potential when all the motor fibres have been eliminated by disease. Unfortunately, we have not been able to demonstrate the contribution of motor fibres in a similar way. For this purpose one would need to examine patients with sensory loss but with full preservation of motor innervation. In his work on the arm, Dawson (1956) was able to study a patient with sensory radicular neuropathy (Case 3 of Ogden, Robert, and Carmichael, 1959); in this patient a nerve action potential with an amplitude of $28 \mu \mathrm{v}$ was recorded from the ulnar nerve above the elbow which was considered to be derived entirely from antidromic impulses set up in motor fibres at the wrist. Recordings from the lateral popliteal nerve in patients with sensory radicular neuropathy have not been carried out, and in the absence of such information the proportion of the action potential which is due to impulses in motor fibres remains uncertain.

These considerations are important in the interpretation of findings from pathological cases. A small action potential with an amplitude below the lower limit of the normal range could be due to a partial lesion of both motor and sensory fibres or to a complete lesion of one with preservation of the other. Thus it is only when the nerve action potential is wholly absent that one may conclude that both motor and sensory fibres are affected. In the present series the nerve action potential has been wholly absent in all the patients with polyneuritis and in a proportion of those with subacute combined degeneration and hereditary disorders such as peroneal muscular atrophy. From the clinical point of view, therefore, these conditions seem to offer the most promising field for further investigation and a study of diabetic neuritis is now being undertaken (Gilliatt and Willison, 1962).

At this point it may be asked what is the nature of the nerve lesion causing diminution or loss of the action potential. Degeneration of some or all of nerve fibres would be expected to have this result but in our patients with pressure palsies of the lateral popliteal nerve recovery was so rapid that degeneration cannot have occurred, and yet abnormalities of the action potential were consistently present. The results in these patients suggest that dispersion of the ascending volley may be an important factor. For example, in the case illustrated in Fig. 9 conduction velocity in the fastest motor fibres to the extensor digitorum brevis was normal, although the nerve action potential was much reduced in size and showed an increased latency to peak. This result is similar to our previous findings in patients with ulnar nerve lesions at the elbow and it is most easily explained by dispersion of impulses in different fibres of the nerve. For this reason the nerve action potential is a more sensitive index of disturbed function than the simple measurement of conduction time for the fastest surviving motor fibres.

Finally, it must be emphasized that the recording of nerve action potentials only provides information about motor fibres and about the larger and more rapidly conducting sensory fibres. For the small afferent fibres there is no satisfactory technique which can be applied to patients at the present time. However, the presence of degeneration in small 
fibres can be inferred from the results of histamine or cold vasodilatation tests (Bonney, 1954), and these techniques can sometimes be of great value in supplementing nerve conduction studies (Bonney and Gilliatt, 1958).

\section{Summary}

When the anterior tibial nerve at the ankle was stimulated with single shocks, it proved possible to record the action potential of the ascending volley from the lateral popliteal nerve at the head of the fibula. Surface and needle recording electrodes were tried, and it was concluded that subcutaneous needles inserted close to the nerve trunk were more satisfactory than surface electrodes placed on the skin over the course of the nerve.

Action potentials were recorded from the lateral popliteal nerve in 39 control subjects; their amplitude varied from 2.0 to $15.5 \mu \mathrm{v}$, the lowest values being seen in elderly subjects. The conduction velocity of the ascending volley was also slightly reduced in the older subjects.

Results in patients with multiple sclerosis did not differ significantly from those of the control group, and in patients with motor neurone disease it was possible to record nerve action potentials even when the motor fibres of the anterior tibial nerve had been eliminated by the disease.
In contrast to the patients with spinal cord disease, nerve action potentials were absent or grossly abnormal in those with polyneuritis or localized lesions of the lateral popliteal nerve. Abnormalities of the action potential were also present in patients with subacute combined degeneration and with peroneal muscular atrophy, but the results were more variable than in polyneuritis.

We wish to thank members of the staff of the National Hospital and of the Middlesex Hospital who have referred patients for investigation.

A personal grant to H.V.G. from the Sir Halley Stewart Trust is gratefully acknowledged. We are also indebted to the Medical Research Council and to the Mary Kinross Charitable Trust for grants defraying the cost of apparatus and to the Clinical Research Committee of the Middlesex Hospital for providing facilities. This work would not have been possible without the technical assistance of Mr. H. B. Morton and Mr. E. E. Dowsett.

\section{REFERENCES}

Bonney, G. (1954). Brain, 77, 588.

Buller, A. J. (1959). Personal Communication. Med., 51, 365.

Dawson, G. D. (1956). J. Physiol. (Lond.), 131, 436.

, and Scott, J. W. (1949). J. Neurol. Neurosurg. Psychiat., 12 259.

Gilliatt, R. W, and Thomas, P. K. (1960), Ibid, 23, 312

, and Willison, R. G. (1962). To be published.

Hunt, C. C. (1954). J. gen. Physiol., 38, 117.

Ogden, T. E., Robert, F., and Carmichael, E. A. (1959). J. Neuro市 Neurosurg. Psychiat., 22, 267.

Thomas, P. K., Sears, T. A., and Gilliatt, R. W. (1959). Ibid, 22, $17 \underset{<}{\sigma}$ 